\title{
Neuroblastoma torácico en la adolescencia. Caso clínico
}

\author{
C. CARIDAD VERDECIA C. ${ }^{1}$, ALFREDO NARANJO U. ${ }^{2}$, KATIA RIVERA L. ${ }^{3}$, LUIS E. MARCANO S. ${ }^{4}$ \\ 1. Especialista de segundo Grado en Oncología y Cirugía Pediátrica. Jefa del Servicio Oncopediatría \\ Hospital Pediátrico Docente William Soler. Cuba. \\ 2. Especialista de segundo Grado en Cirugía Cardiovascular. Cardicentro William Soler. \\ 3 Residente de Cirugía Cardiovascular. Cardicentro William Soler. \\ 4. Especialista de segundo Grado en Cirugía Cardiovascular. Cardicentro William Soler.
}

\begin{abstract}
Thoracic neuroblastoma in adolescence. Clinical case

Neuroblastoma is predominantly a tumor of early childhood, most cases occur in children under 5 years old. It originates in the adrenal gland and paravertebral ganglion cells (neural crest-derived), being the most common an extracranial solid tumor in children. It is characteristic a spontaneous regression, However in some cases it shows progression and dissemination to other organs. Objetive: To show a neuroblastoma in adolescence, with poor response to chemotherapy and radiotherapy, requiring surgery treatment. Clinical case: A 16 y.o. Female patient, previously asymptomatic, who after a body temperature rise up to $39^{\circ} \mathrm{C}$, was found to have a tumor in the right hemithorax. Biopsy was compatible with neuroblastoma. Surgical removal of a large 20 x $19 \mathrm{~cm}$ tumor was achieved, the only complication presented 10 days postop was recurrent pneumothorax. Conclusion: Despite little or no response to standard treatment, surgical resection of this large tumor achieved complete remission for this patient.
\end{abstract}

(Key words: Neuroblastoma chest, adolescence, ganglion cells, suprarrenal).

Rev Chil Pediatr 2012; 83 (2): 170-174

\section{RESUMEN}

El neuroblastoma es predominantemente un tumor de la infancia temprana que en la mayoría de los casos se presenta en menores de 5 años. Se origina en la glándula suprarrenal y células ganglionares paravertebrales (derivadas de la cresta neural), siendo el tumor sólido extracraneal más común en pediatria, presenta regresión espontánea en algunos casos y en otros progresión y diseminación a otros órganos. Objetivo: Analizar el caso de una adolescente portadora de un neuroblastoma resistente a quimioterapia y radioterapia, que requiere tratamiento quirurgico. Caso clínico: Paciente asintomatica, edad 16 años, que a raíz de alza febril se le detecta un tumor en hemitórax derecho. La biopsia fue compatible con un neuroblastoma. Se realizo la extirpación quirúrgica de un gran tumor con diámetro de $20 \mathrm{~cm}$ x $19 \mathrm{~cm}$. A los 10 días se complica con neumotórax recidivante. Conclusión: A pesar de la poca o nula respuesta a las armas terapéuticas habituales: quimioterapia y radioterapia, la cirugía logró la resección del tumor torácico con remisión completa.

(Palabras clave: Neuroblastoma torácico, adolescencia, suprarrenal, ganglios).

Rev Chil Pediatr 2012; 83 (2): 170-174

Trabajo recibido el 11 de noviembre de 2011, devuelto para corregir el 15 de diciembre de 2011, segunda versión 26 de enero de 2011, aceptado para publicación el 03 de febrero de 2012.

Correspondencia a:

Dra. C. Caridad Verdecia Cañizares

E-mail: caryverd infomed.sld.cu 


\section{Introducción}

El neuroblastoma es el tumor sólido extracraneal más frecuente en el niño, el cual deriva de las células de la cresta neural y médula suprarrenal.

La mayoría de los casos se presentan en menores de 5 años de edad. Representa entre el 8 y el $10 \%$ de todos los tumores pediátricos, su incidencia global es de 9,9 x 10000000 habitantes (550 casos nuevos por año en EE UU). En Cuba se diagnostican cada año entre 8 y 10 casos nuevos, siendo la edad media de presentación 22 meses ${ }^{1}$. Aproximadamente el $36 \%$ de los casos se diagnostica antes del año de edad, el $79 \%$ antes de los 4 años y el $97 \%$ antes de los 10 años, siendo un tumor altamente maligno y con una sobrevida global de un $50 \%$ a nivel mundial ${ }^{2,3}$. Es característica la regresión espontánea en algunos casos y la progresión tumoral en otros con diseminación temprana a otros órganos ${ }^{2,3}$.

La ubicación más frecuente es el abdomen, en la glándula suprarrenal en un $70 \%$, pero un $20 \%$ se originan en el tórax, siendo éstos de mejor pronóstico. Los síntomas más comunes se deben a la compresión por la masa tumoral o al dolor óseo causado por la metástasis, siendo frecuente el compromiso del estado general, la pérdida de peso, la astenia y la anorexia. La proptosis y la equimosis periorbital se deben a las metástasis retrobulbares. La infiltración de la médula ósea podría causar pancitopenia. En el lactante es frecuente la distensión abdominal con dificultad respiratoria debido a metástasis hepáticas masivas. Aquellos originados en los ganglios paraespinales, pueden invadir los agujeros neurales, comprimir la médula espinal y causar parálisis. Ocasionalmente se pueden presentar por anemia e hipertensión. El neuroblastoma se puede presentar con diarrea grave debido a que el tumor secreta péptido intestinal vasoactivo, y también manifestarse por fiebre ${ }^{2,4-6}$.

El objetivo del trabajo es dar a conocer un caso clínico de neuroblastoma en la adolescencia.

\section{Caso clínico}

Paciente de sexo femenino de 16 años, caucásica, consulta por fiebre de $39^{\circ} \mathrm{C}$. En la radiografía de tórax se detecta un gran tumor torácico derecho. El estado clínico era conservado y al examen físico no se pesquizó ninguna alteración.

\section{Estudios realizados}

Se efectuó una radiografía de tórax que mostró una opacidad que abarcaba los 2/3 inferiores del hemitórax derecho con calcificaciones en su interior.

El ultrasonido torácico mostró un tumor sólido de bordes bien definidos, en íntima relación con hemidiafragma derecho, sin infiltración, de $109 \mathrm{~mm}$ x $95 \mathrm{~mm}$ de diámetro, con un pequeño derrame pleural derecho y múltiples calcificaciones en su interior. Ultrasonido de abdomen: hígado, bazo, páncreas, riñones normales, asas intestinales normales y ambas suprarrenales de apecto normal.

La TAC de tórax mostró una imagen de densidad tumoral sólida, de 107 x 97 mm con calcificaciones, muy vascularizada con captación de contraste endovenoso, que comprime y desplaza estructuras vasculares vecinas del mediastino, comprime el pulmón derecho hacia arriba y desplaza hemidiafragma hacia abajo, con múltiples calcificaciones (figura 1).

Hemograma y coagulación: normales. Deshidrogenasa láctea (LDH): elevada 778 UI. (Valor normal: 200-400 UI). Estudios de función hepática y renal normales, glicemia normal. Proteínas totales y albúmina: normales. Determinación de ácido vanil mandélico (AVM) estaba en cifras normales. Se realizó biopsia aspirativa con aguja fina (BAAF) bajo control ultrasonográfico obteniendo muestra para estudio citológico que arrojó positivo para malignidad, pero sin poder tipificar variedad del tumor. Medulograma mostró los 3 sistemas íntegros, sin infiltración medular. Biopsia Médula ósea: negativa para infiltración tumoral. Biopsia quirúrgica del tumor: Neuroblastoma indiferenciado, $\mathrm{N}$-myc amplificado con más de 10 copias.

Se inició tratamiento con quimioterapia para reducción del volumen tumoral con OPEC/OJEC con descanso de 21 días entre los ciclos con dosis habituales (OJEC: Vincristina, Carboplatino, Ciclofosfamida, Etopóxido) (OPEC: Vincristina, Cisplatino, Etopóxido, 


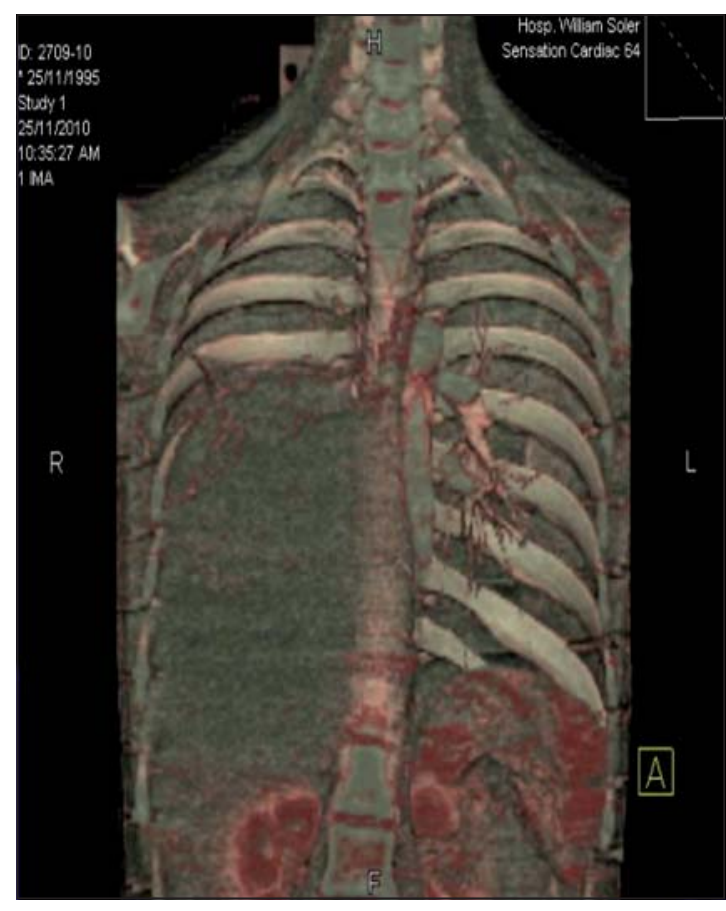

Figura 1. TAC contrastada de tórax antes de la cirugía con técnica de extracción ósea para ver bien el tumor localizado hacia la derecha.

Ciclofosfamida). Recibió 3 ciclos completos de cada uno y la evaluación radiológica mostró una progresión tumoral. Se realizó radioterapia a dosis total de 6070 cGy, sin respuesta. Se discutió en equipo multidisciplinario y se planteó la cirugía como terapia de salvataje, con alto riesgo por la compresión de estructuras vitales del mediastino. Se realizó toracotomía posteroláteral derecha ampliada, encontrando gran tumor, íntimamente adherido al hemidiafragma, a la vena cava superior y al pericardio, se logra separar de éstas estructuras ligando gran cantidad de vasos de neoformación (figura 2). La recuperación postoperatoria fue buena, no requiriendo ventilación mecánica, el drenaje pleural drenó escasa cantidad de líquido serohematico, siendo retirado a las 48 horas de la cirugía. Presentó como complicación neumotórax espontáneo a los 10 días y hemoneumotórax tabicado, el cual se resolvió con limpieza de la cavidad pleural, logrando gran re-expansión pulmonar derecha. Actualmente, la paciente esta ambulatoria con seguimiento

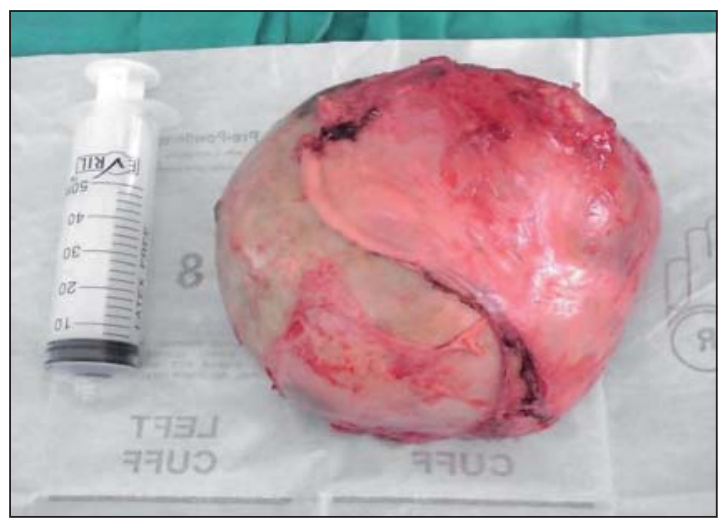

Figura 2. Tumor gigante resecado quirúrgicamente de 20 x $19 \mathrm{~cm}$.

de 11 meses. El resultado de anatomía patológica reveló un Neuroblastoma moderadamente diferenciado, bien encapsulado, con áreas de necrosis central, bordes de sección libres de tumor residual y que medía aproximadamente 20 X19 centímetros, N- myc con amplificación de más de 10 copias.

\section{Discusión}

El NB torácico es el tumor neurogénico más común del mediastino ${ }^{2,7,8}$, aunque infrecuente en la adolescencia se han reportado varios casos en la literatura, existen estudios que plantean la regresión espontánea en ésta localización. Demir y cols, del departamento de Oncología Pediatría del Instituto Oncología de Ankara, Turquía, publicaron en la revista Bood and Cancer una serie de 638 pacientes con NB torácico primario diagnosticados entre el año 1973 y 2007 de los cuáles 87 niños fueron elegibles, 51 sexo femenino y 36 del masculino, con una edad media de los pacientes de 2,1 años y una sobrevida a los 10 años de un 71,2\%. Las manifestaciones clínicas que presentaron fueron síntomas respiratorios, dolor torácico, manifestaciones de compresión neurológica (paraplejia), fiebre, síndrome de Horner y síndrome de opsoclono mioclonuos ${ }^{9}$. Existiendo un consenso de que el neuroblastoma torácico pediátrico tiene un pronóstico favorable y muchos tienen regresión espontánea ${ }^{2,3}$. 
Morris y cols, reportaron 227 casos de neuroblastoma torácico con una buena evolución y tiempo de sobrevida elevado independientemente de factores clínicos y variables biológicas como niveles en suero elevado de la LDH, ferritina y MYCN encogen ${ }^{8,9}$. Otros autores plantean que se han identificado eventos de regresión espontánea en etapas tempranas de la infancia ${ }^{4,5,7-9}$. Schiling y cols, suponen que esta hipótesis puede encontrarse en NB torácicos incompletamente resecados, el sólo encontró recurrencia local en un paciente de 13 casos operados $^{8}$. Adams y cols, en 96 pacientes con NB torácico, 49\% no tenían manifestaciones clínicas, estaban totalmente asintomáticos, mientras que el 19\% tenía paraplejia por compresión medular causada por el tumor y un 3\% síndrome de Horner al debut ${ }^{10}$. En nuestro caso se plantea que el crecimiento lento, paulatino de éste tumor hizo que se detectara fortuitamente debido a una hipertermia, sin otras manifestaciones.

La quimioterapia adyuvante antes de la cirugía es empleada para reducir los grandes volúmenes tumorales en casos avanzados como parte de la terapia multimodal, continuando con la misma asociada o no a la radioterapia sobre todo si es imposible la resección completa del tumor o ya existe extensión a otras estructuras, con lo cual se ha logrado elevar la sobrevida $^{9-11}$.

En nuestro país se utilizan iguales esquemas internacionales de grupos cooperativos y nacionales para el tratamiento de éstos tumores, el Dr. Alert y cols ${ }^{13}$, han publicado trabajos del empleo de la radioterapia junto a la quimioterapia adyuvante para el tratamiento de diversos tumores pediátricos como es el NB con muy buenos resultados en etapas clínicas avanzadas de la enfermedad. El más efectivo protocolo para el tratamiento del NB no ha sido bien establecido aún, porque a pesar de los esfuerzos que se realizan por los equipos multidisciplinarios y esquemas multidrogas la sobrevida actual global es de un 50\% en el NB avanzado $^{8-13}$.

El NB torácico estadio avanzado (etapas III y IV) después de una quimioterapia intensa y radioterapia, aunque no se haya podido resecar completamente el tumor, muestran ma- duración espontánea a ganglioneuroblastoma y ganglioneuroma detectados en la segunda cirugía ${ }^{2,13-15}$. Muchos estudios reportan la alta sobrevida de los casos de NB torácico comparados con otros sitios anatómicos como por ejemplo el abdominal, aunque no se conoce la razón exacta; se plantean muchos factores como son los factores inmunes, manifestaciones clínicas tempranas dada por la compresión de las estructuras mediatinales, número de copias del N-MYC oncogen, edad temprana de aparición y características biológicas del tumor en ésta localización ${ }^{1,9,14-17}$.

Como conclusión podemos decir que la experiencia alcanzada reafirma el valor del manejo en equipo multidisciplinario en el diagnóstico, tratamiento y rehabilitación de los pacientes. La decisión terapéutica debe individualizarse en cada caso dependiendo de la respuesta al tratamiento, para lograr el mejor resultado. A pesar de la poca o nula respuesta a las armas terapéuticas habituales: quimioterapia y la radioterapia en nuestra paciente, la cirugía que aunque riesgosa logró la resección del gran tumor torácico con remisión completa.

\section{Referencias}

1.- Martin A, Alert J, Renó J, Longchong M, Grueiro S: Incidence of childhood cancer in Cuba(1986-1990). Int J Cancer 1997; 72: 551-5.

2.- Horiuchi A, Muraji T, Tsugawa C: Thoracic neuroblastoma: Outcome of incomplete resection. Pediatric Surg Int 2004; 20: 714-8.

3.- Habele B, Hero B, Berthold F: Characteristics and outcome of thoracic neuroblastoma. Eur J Pediatr Surg 2002; 12: 145-50.

4.- Spitz R, Hero B, Simon T: Loss in chromosome $11 \mathrm{q}$ identifies tumors with increased risk for metastatic relapses in localized and 4S neuroblastoma. Clin Cancer Res 2006; 12 (11 Pt 1): 3368-73.

5.- $\quad$ Ohali A, Avigad S, Ash S: Telomere length is a prognostic factor in neuroblastoma. Cancer 2006; 107: 1391-9.

6.- Wei JS, Greer BT, Westermann F: Prediction of clinical outcome using gene expression profiling and artificial neural networks for patients with neuroblastoma. Cancer Res 2004; 64: 6883-91.

7.- Caron HN: Are thoracic neuroblastomas really diffe- 
rent? Pediatric Blood Cancer 2010; 54: 867.

8.- Schilling FH, Spix C, Berthold F: Neuroblastoma screening at one year of age. N Engl J Med 2002; 346: 1047-53.

9.- Demir HA, Yelçin B, Nebil B, Gulsev K: Thoracic neuroblastic tumors in childhood. Pedatric Blood and Cancer 2010; 54: 885-9.

10.- Adams GA, Shochat SJ, Smith EI: Thoracic neuroblastoma: A pediatric oncology group study. J Pedaitr Surg 1993; 28: 372-8.

11.- Yamamoto K, Ohta S, Ito E: Marginal decrease in mortality and marked increase in incidence as a result of neuroblastoma screening at 6 months of age: cohort study in seven prefectures in Japan. J Clin Oncol 2002; 20: 1209-14.

12.- Okazaki T, Kohno S, Mimaya J: Neuroblastoma detected by mass screening: the Tumor Board's role in its treatment. Pediatr Surg Int 2004; 20: 27-32.

13.- Alert J: Empleo del tratamiento radiante en los tumores de cabeza y cuello en el niño. Rev Cubana Oncol 2001; 17: 200-9.

14.- Park JR, Eggert AE, Caron HN: Neuroblastoma: Biology, prognosis and treatmen. Pediatr Clin N Am 2008; 55: 97-120.

15.- Horiuchi A, Muraji T, Tusugawa C: Thoracic neuroblastoma: Outcome of incomplete resection. Pediatr Surg Ins 2004; 20: 714-8.

16.- Kitanaka C, Kato K, Ijiri R: Increased Ras expression and caspase-independent neuroblastoma cell death: possible mechanism of spontaneous neuroblastoma regression. J Natl Cancer Inst 2002; 94: 358-68.

17.- Takeda S, Miyoschi S, Minami M: Intrathoracic neurogenic tomors: 50 years experience in a Japanaise institution. Eur J Cardiothorac Surg 2004; 26: 807-12. 